\title{
Damage evolution in the AlMg6 alloy during high and very high cycle fatigue
}

\author{
Mikhail Bannikov, Dmitry Bilalov, Vladimir Oborin, Oleg Naimark \\ Institute of Continuous Media Mechanics UB RAS, Russia \\ mbannikov@icmm.ru, bttp:/ / orcid.org/0000-0002-5737-1422 \\ ledon@icmm.ru,bttp:/ /orcid.org/0000-0003-1541-2246 \\ oborin@icmm.ru,https:/ / orcid.org/0000-0003-2836-2073 \\ naimark@icmm.ru, bttps://orcid.org/0000-0001-6537-1177
}

\begin{abstract}
Paper presents the "in situ" method for determining of irreversible fatigue damage accumulation, based on the analysis of nonlinear manifestations of the feedback signal in a closed system of an ultrasonic fatigue system. During very high cycle (gigacycle) fatigue, the anomalies of the elastic properties of the material are appear, which leads to a nonlinearity effect in the amplitude of oscillations. This effect increases with the initiation and growth of fatigue cracks. The technology was applied to samples of AlMG-6 alloy with preliminary dynamic deformation to determine the moment of initiation and growth of the fatigue crack in very high cycle fatigue regime. This method is applicable for the early detection of fatigue cracks both on the surface and inside the material under cyclic loading in the ultrasonic mode. On the basis of wide-range defining relations for a deformable solid body with mesoscopic defects, a mathematical model has been proposed that can adequately describe behavior of the material during fatigue failure. The results of mathematical modeling are in good agreement with the experimental data
\end{abstract}

KEYwORDS. Damage accumulation; Scaling; High- and very high cycle fatigue; Mathematical modeling; Destruction, mesoscopic defects.

\section{OPEN ACCESS}

Citation: Bannikov, M., Bilalov, D., Oborin, V., Naimark, O., Damage evolution in the AlMg6 alloy during high and very high cycle fatigue, Frattura ed Integrità Strutturale, 49 (2019) $383-395$.

Received: 01.04.2019

Accepted: 14.06 .2019

Published: 01.07.2019

Copyright: (C) 2019 This is an open access article under the terms of the CC-BY 4.0, which permits unrestricted use, distribution, and reproduction in any medium, provided the original author and source are credited.

\section{INTRODUCTION}

$\mathrm{T}$ he determination of fatigue cracks origin nature is one of the most important fundamental problem for various application areas, especially speaking about ultrahigh-cycle (gigacycle) [1-3] fatigue, when crack forms inside the material. The series of catastrophes caused by the fatigue destruction of gas turbine engines [4-6], combined with the high cost of resource assessment and the potential cost of developing new structures, stimulated promising concepts of national programs in the field of high and very high cycle fatigue, based on the use of new fundamental results in 
assessing fatigue strength. Special attention should be paid to the foreign object damage (FOD) research section, which deals with the destruction of engine parts after preliminary dynamic loading [7-11].

At present, after several decades of research, it is becoming clear that fatigue damage is the result of accumulation of microplastic deformations, which lead to irreversible microstructural and topological changes [12] that determine critical states of damage and the transition to destruction. General knowledge of the mechanisms of microstructural changes leading to fatigue failure allows us to associate these critical states with the development of an ensemble of microcracks originating in localized shifts.

The interest to fundamental problems of fatigue damage has sharply increased in connection with the possibility of attaining a fatigue resource corresponding to the so-called very high (gigacycle) fatigue [1-10]. The results of these studies raised, in particular, the question of the existence of a fatigue limit in the transition from high cycle to gigacycle loading conditions.

The formation of fatigue damage is traditionally associated with microplastic deformations [1-2, 12], which are occur under cyclic loading conditions, initiating various microstructural mechanisms that control durability and depend on the nature and initial structure of the material. For ductile metals, a special type of fatigue (cyclic) deformation localization (persistent slip bands - PSB) is observed, the development of which traditionally leads to the initiation of cracks in the near-surface zone. The second common case, for example, in the fatigue fracture of high-strength steels, is the onset of damage (microcracks), in the vicinity of inclusions at low load amplitudes corresponding to very high cycle fatigue.

A characteristic feature of destruction under gigacycle fatigue conditions is the decisive effect of the initiation stage of a fatigue crack on the fatigue life. At the same time, a qualitative difference is the formation of a fatigue crack in the bulk of the material, which decisively changes the formulation of the problem of assessing the fatigue life, methods for studying the stages of fracture development. In contrast to the established traditions in the field of high-cycle fatigue, where central attention is paid to the crack propagation stage, a fundamental problem arises about formation of the fatigue crack during multiscale damage development processes associated with defects of various nature (inclusions, localized plastic shear bands, microcracks, pores). The staging of destruction is characterized by the effects of "irreversibility", initiated by the formation of localized shifts that play a key role in the initiation of a fatigue crack [13-15], which can manifest itself in signs of nonlinearity of the elastic behavior of materials, "anomalies of elastic compliance" of fatigue samples. The role of the initiation stage is especially important for gigacyclic loading regimes, which are characterized by "fish-eye" crack formed in the bulk of the material [13-14]. This article presents a method for monitoring the kinetics of accumulation of defects by analyzing the amplitude of the second harmonic of oscillations of the free end of the sample. With the appearance of internal defects, nonlinearity effects arise, which significantly increase with their correlated interaction and the formation of a fatigue crack.

\section{MATERIAL AND EXPERIMENT CONDITION}

I $\mathrm{n}$ this research were investigated samples of aluminum alloy AMG-6, the chemical composition and mechanical characteristics of which are presented in Tabs. 1 and 2.

The material was subjected to dynamic deformation performed by split Hopkinson pressure bar set-up at the strain rates of $\sim 10^{3} \mathrm{~s}^{-1}[16]$. The material was pre-deformed in order to specify a certain degree of initial damage to assess further of the defects development kinetics. Samples with a geometry corresponding to Fig. 1.

\begin{tabular}{ccccccccc}
\hline $\mathrm{Al}$ & $\mathrm{Cu}$ & $\mathrm{Mg}$ & $\mathrm{Mn}$ & $\mathrm{Si}$ & $\mathrm{Fe}$ & $\mathrm{Zn}$ & $\mathrm{Be}$ & $\mathrm{Ti}$ \\
\hline $91.1-93.68$ & 0.10 & $5.8-6.8$ & $0.5-0.8$ & 0.4 & 0.4 & 0.20 & $0.0002-0.005$ & $0.02-0.1$ \\
\hline
\end{tabular}

Table 1: The chemical composition of AMg6 (as a percentage)

Elastic modulus (GPa) Yield strength (MPa) Tensile strength (MPa) Maximum elongation (\%) 71 180 355 


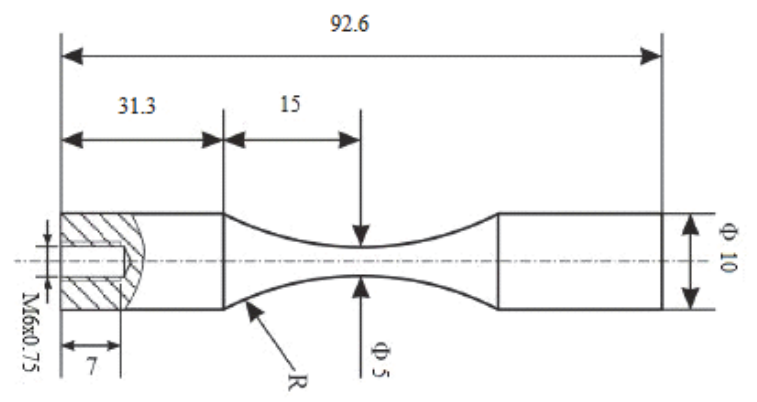

Figure1: Geometry of the samples (scales in $\mathrm{mm}$ )

The fatigue loading was carried out on an ultrasonic Shimadzu USF-2000 resonant-type testing machine (Fig. 2) at stress levels of $90-162 \mathrm{MPa}$ and a symmetrical cycle $\mathrm{R}=-1$. The principle of operation testing machine is based on formation of standing wave in the generator-horn-sample system [2]. In this case, the maximum amplitude of displacements occurs at the ends of the sample, and the maximum amplitude of stresses at its center. On the side of the free end of the specimen, an inductive sensor is fixed, which makes possible to determine the displacement of the end with an accuracy of tenths of a micron and a frequency of up to $1 \mathrm{MHz}$. The machine allows testing materials on the basis of $10^{9}-10^{10} \mathrm{cycles}$ with amplitude from the 1 st to several tens of microns with a frequency of $20 \mathrm{kHz}$, which reduces the test time to several days, in contrast to the classical fatigue installations, where this number of cycles is achieved during the years of testing.

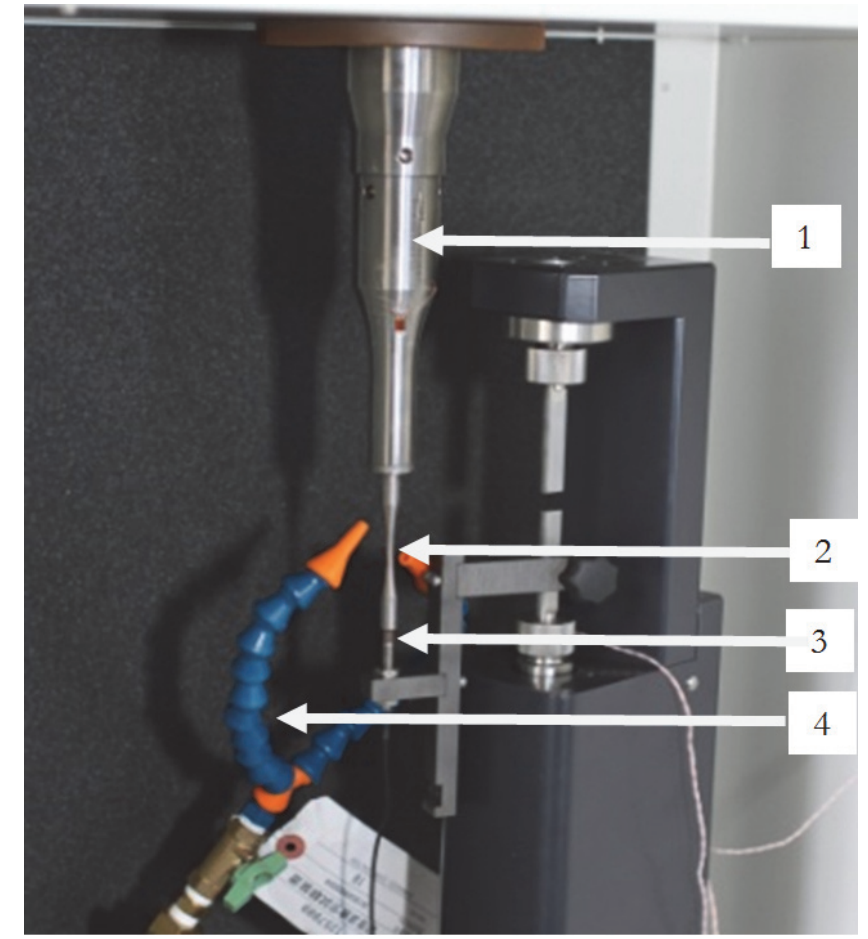

Figure 2: The experimental setup: 1 - Horn, 2 - sample, 3 - displacement sensor, 4 - cooling system.

The formation of cracks in cylindrical samples undergoing high cycle loading in the range $10^{6}-10^{7}$ cycles begins from the surface. For the dynamic preloading samples AlMg6 subject to cyclic loads in the range exceeding $10^{8}$ cycles the crack formation is started in the bulk of the sample. The fracture surface in this case exhibits a fatigue zone known as a "fisheye" which is a distinguishing feature of such fatigue regimes. The central part of this region comprises a fracture nucleus surrounded by the region of refined (submicrocrystalline) structure so-called FGA [13] (Fig. 3a). 


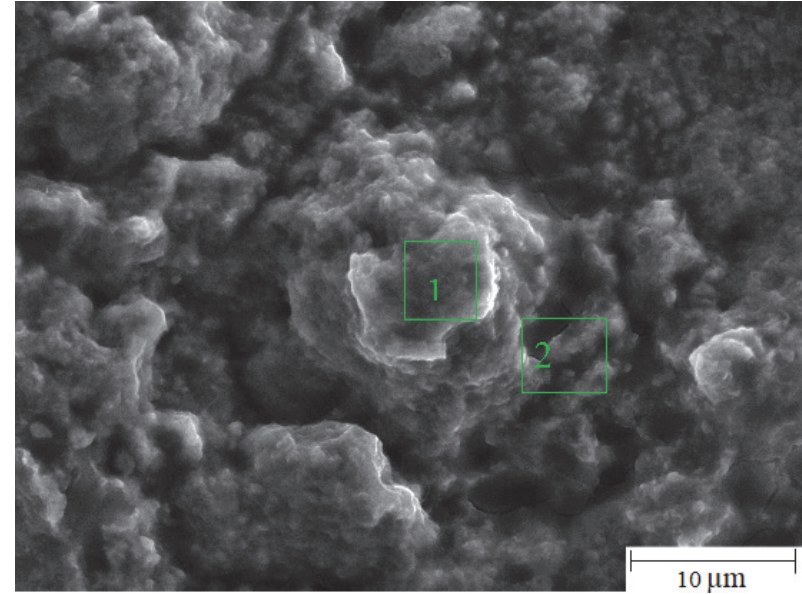

a)

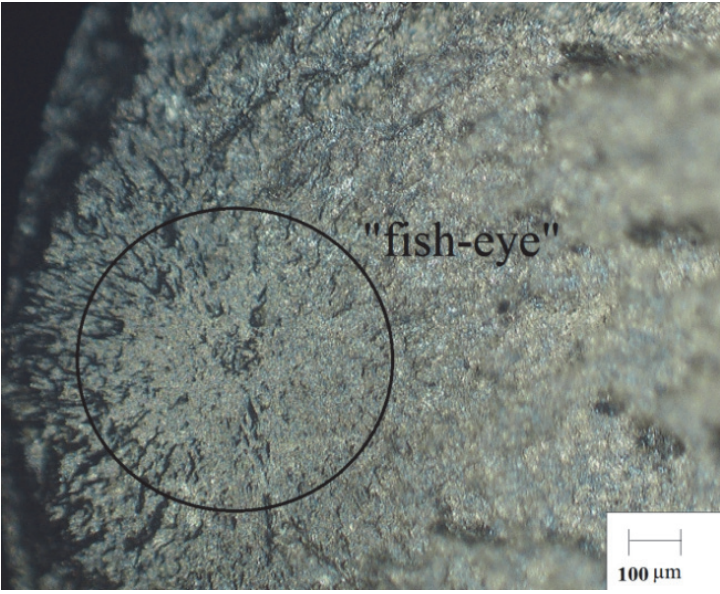

(b)

Figure 3: (a) Characteristic surface relief of a gigacycle fatigue fracture zone of AlMg6, (b) scaled-up fragment of the «fish-eye» zone obtained by the scanning electron microscope Hitachi $\mathrm{S} 3400\left(\sigma=138 \mathrm{MPa} \mathrm{Nf}=7.51 \cdot 10^{8}\right)$, the region 1 is the center of crack initiation, the region 2 is FGA zone.

\section{NONLINEAR ACOUSTICS METHOD FOR NON-DESTRUCTIVE TESTING OF FATIGUE FAILURE}

7 he method is based on the initiation of a longitudinal finite-amplitude perturbation $A_{0}$ with a frequency $\omega_{0}$ on one side of the sample, while its other end remains free [17-19]. Oscillations of the free end of the sample will contain a number of harmonic components: components with amplitude $A_{1}$ at the fundamental frequency $\omega_{0}$, amplitude $\mathrm{A}_{2}$ of the second harmonic with frequency $2 \omega_{0}$, and so on. The nonlinearity parameter $\beta^{e}$ is determined experimentally by measuring the absolute amplitudes of the signals of the first $A 1$ and second $A_{2}$ harmonics corresponding to the nonlinear law of elasticity:

$$
\sigma=A_{2}^{e}\left(\frac{\partial u}{\partial a}\right)+\left(\frac{1}{2}\right) A_{3}^{e}\left(\frac{\partial u}{\partial a}\right)^{2}+\ldots=A_{2}^{e}\left[\left(\frac{\partial u}{\partial a}\right)-\frac{1}{2} \beta^{e}\left(\frac{\partial u}{\partial a}\right)^{2}+\ldots\right]
$$

where $\sigma$ is the stress, $u$ is the displacement, $a$ is the spatial coordinate, $A_{2}^{e}$ and $A_{3}^{e}$-are the second and third order elastic coefficients, respectively. By introducing the nonlinearity coefficient: $\beta^{e}=-\left(A_{3}^{e} / A_{2}^{e}\right)$ and the wave equation can be represented as:

$$
\frac{\partial^{2} u}{\partial t^{2}}=c^{2}\left[1-\beta^{e}\left(\frac{\partial u}{\partial a}\right)\right]\left(\frac{\partial u}{\partial a}\right)
$$

where $\mathrm{u}$ is the component of the displacement vector in the direction, $c$ - is the longitudinal speed of sound, $t$ - is time. Its solution, given that the end oscillation $u=u 1 \cos (\omega t)$, will be:

$$
u=u_{0}+u_{1} \cos (\omega t)+u_{2} \sin 2(\omega t-k a)+\ldots
$$

That the amplitude of the second harmonic $u_{2}=(1 / 8) k^{2} \beta^{e} u_{1}^{2} a$, where $k=\omega_{0} / v-$ wave number, we can derive:

$$
\beta^{e}=8 u_{2} / k^{2} u_{1}^{2} a
$$

In the study of nonlinear phenomena in the gigaclic fatigue regime, the relative parameter is determined by measuring the amplitudes of the main and second harmonics: 


$$
\beta_{\text {relative }}=\beta / \beta_{0}
$$

where $\beta_{0}$ refers to intact material.

\section{MEASUREMENT OF NONLINEARITY COEFFICIENT}

o determine the nonlinearity coefficient, we prologue both parts of the Eqn. (4):

$$
\log (\beta)=\log (k)+\log \left(u_{2}\right)-2 \log \left(u_{1}\right)
$$

The amplitude of the oscillations is measured in decibels at scales $A 1=20 \log (u 1)$ and $A 2=20 \log (u 2)$, then the ratio takes the form:

$$
\begin{aligned}
& 20 \log (\beta)=20 \log (k)+20 \log \left(u_{2}\right)-40 \log \left(u_{1}\right), \\
& 20 \log (\beta)=K+A_{2}-2 A_{1}
\end{aligned}
$$

Thus, the relative nonlinearity parameter $\beta$ can be found from the formula:

$$
20 \log \left(\beta / \beta_{0}\right)=\left(A_{2}-2 A_{1}\right)-\left(A_{2}-2 A_{1}\right)_{0}
$$

The authors developed a software and hardware complex which analyzes the signal in real time with a variable duration and performs a fast Fourier transform to search for the amplitudes of the fundamental and second harmonics. When a trend is detected to increase the amplitude of the second harmonic, the software stops the experiment and reports the possible occurrence of a fatigue crack.

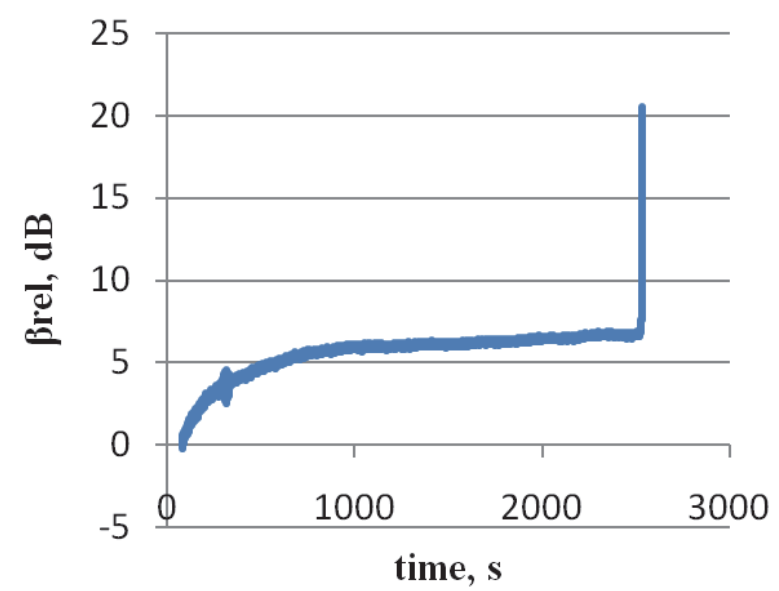

a)

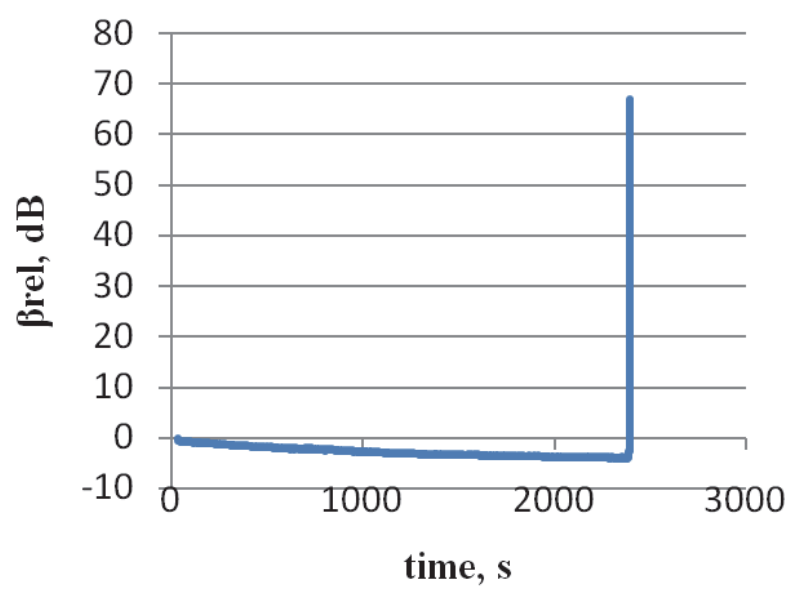

b)

Figure 4: The change in the non-linearity factor $\beta$ relative $(\mathrm{dB})$ during testing of the sample with an internal crack $(\mathrm{a}) \sigma=138 \mathrm{MPa}$, $\mathrm{N}_{\mathrm{f}}=7.51 \cdot 10^{8}$ (Fish-eye) and a sample with a surface crack (b) $\sigma=140 \mathrm{MPa}, \mathrm{N}_{\mathrm{f}}=2.72 \cdot 10^{8}$.

The dependences of the non-linearity coefficient $\beta$ relative on the loading amplitude during fatigue life for aluminum alloy

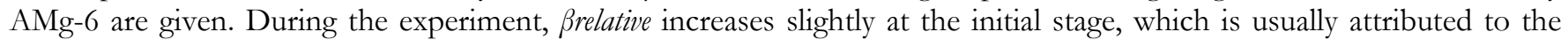
effect of heating the sample and, as a result, changing its linear dimensions [17-19], then most of the time of the experiment, the amplitude of the second harmonic remains constant and increases significantly with the formation of a fatigue crack and its growth (Fig. 4, a). The amplitude of the second harmonic is associated with the deformation due to defects. For given loading parameters, the system is macroscopically in the elastic region, and all deformations are 
reversible. The defects weakly correlate with each other, and the amplitude of the second harmonic is small and varies little. However, in the process of cyclic loading, irreversible microdamages are appeared, which begin to interact with each other, which subsequently leads to the formation of a fatigue crack.

In one of the samples, which were destroyed with the formation of a surface crack, a monotonic decrease in the amplitude of the second harmonic uncharacteristic for others was found during the testing process (Fig. 4.b). This is supposed to be due to the fact that the initial structure of the material was saturated with defects, since the samples were pre-deformed. During cyclic loading, a small amplitude in the material resulted in relaxation of internal stresses [20], which reduced the nonlinearity of the signal. When a stress relaxation resource is depleted, an avalanche-like growth of the second harmonic amplitude is observed, which indicates a high correlation of defects and their interaction on a large scale compared with the internal nucleation of a fatigue crack.

In the fig. 5 the growth of the amplitude of the second harmonic is shown. At the time point of $5.415 * 10 \wedge 4$, the software stopped the experiment on the basis of a significant increase in the amplitude of the second harmonic ( 6 times), which indicated the formation of a fatigue crack. Later, the experiment was started again, which allowed us to observe

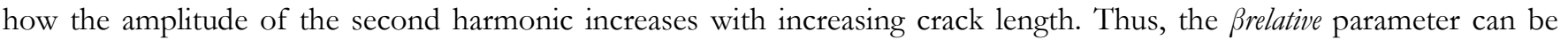
used not only as an indicator of the appearance of a fatigue crack, but also to determine its size, as it was done in [21].

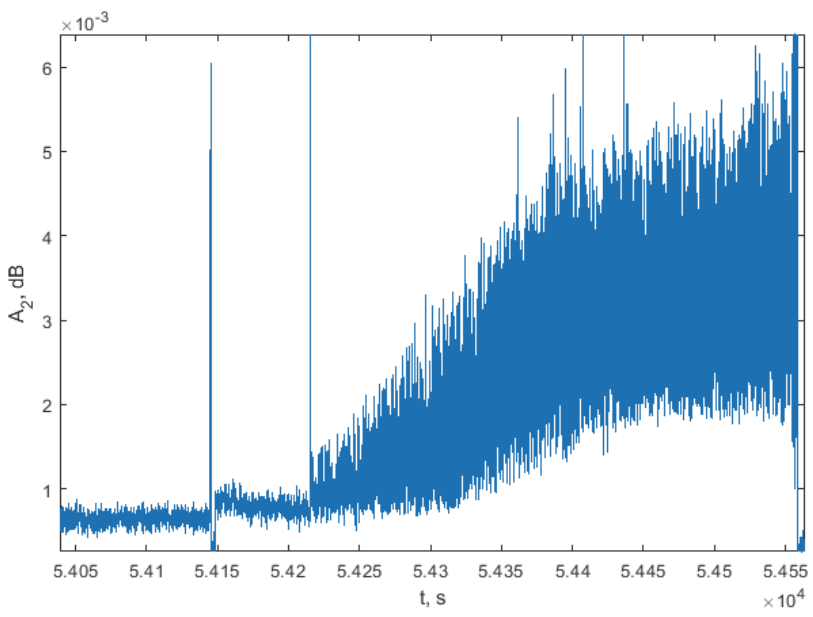

Figure 5. The amplitude of the second harmonic of prestrained sample AMG-6. The difference in the number of cycles between the moment of detection of the nucleation of a fatigue crack and its exit to macroscopic destruction (the output of the system from resonance) was more than $2 \cdot 10^{6}$ cycles.

\section{MATHEMATICAL MODEL}

7 he wide-range constitutive equations [22-24], describing the relationship between the kinetics of damage caused by defects and the relaxation properties of materials, were used to construct a mathematical model that is capable of describing the deformation behavior of metals and alloys under fatigue loading.

To describe the evolution of the structure of the material, two parameters are introduced: tensor:

$$
\mathbf{s}=\frac{s}{2}(\mathbf{l} \mathbf{b}+\mathbf{b} \mathbf{l})
$$

and scalar:

$$
\delta=\left(\frac{R}{r_{0}}\right)^{3}
$$

where: $\mathbf{s}$ - tensor of microshears [23]; $s$ - shear intensity; $\mathbf{1}$ - unit normal vector to the shear plane; $\mathbf{b}$ - unit vector in the direction of shear; $\delta$ - scale-invariant, structural parameter; $R$ - the distance between defects, $r_{0}$ - the characteristic size of the nuclei of defects. 
Averaging s over an elementary volume gives a tensor of microshears density [23]:

$$
\mathbf{p}=\int \mathbf{s} W(s, \mathbf{1}, \mathbf{b}) d V
$$

where: $V$ - volume, $W(s, \mathbf{1}, \mathbf{b})$ - distribution function of orientation and intensity of microshears. In its physical meaning $\mathbf{p}$ is a strain due to defects.

The total strain rate $(\dot{\boldsymbol{\varepsilon}})$ consists of three components: plastic $\left(\dot{\boldsymbol{\varepsilon}}^{p}\right)$, elastic $\left(\dot{\boldsymbol{\varepsilon}}^{e}\right)$ and due to defects $(\dot{\mathbf{p}})$ :

$$
\dot{\varepsilon}=\dot{\varepsilon}^{e}+\dot{\varepsilon}^{p}+\dot{\mathbf{p}}
$$

From the second law of thermodynamics, it follows that the energy dissipation can be represented as:

$$
T \dot{S}=\sigma: \dot{\boldsymbol{\varepsilon}}^{p}-\frac{\partial F}{\partial \mathbf{p}}: \dot{\mathbf{p}}-\frac{\partial F}{\partial \delta} \dot{\delta} \geq 0,
$$

where: $T$ - temperature; $\dot{S}$ - rate of change of entropy; $\sigma$ - stress tensor; - no equilibrium free energy.

According to the Onsager principle, ehe following relations are obtained from (11):

$$
\begin{aligned}
& \sigma=l_{1} \dot{\boldsymbol{\varepsilon}}^{p}-l_{2} \dot{\mathbf{p}} \\
& -\frac{\partial F}{\partial \mathbf{p}}=-l_{2} \dot{\boldsymbol{\varepsilon}}^{p}+l_{3} \dot{\mathbf{p}} \\
& -\frac{\partial F}{\partial \delta}=l_{4} \dot{\delta}
\end{aligned}
$$

where: $l_{1}, l_{2}, l_{3}, l_{4}$ - kinetic coefficients, in the general case, depending on state parameters, satisfying the constraint: $l_{1} l_{3}-l_{2}^{2}>0$.

Relations (12)-(14) are complemented by Hooke's law in the rate formulation and approximation for non-equilibrium free energy. Thus, the complete system of constitutive equations looks as follows:

$$
\begin{aligned}
& \dot{\boldsymbol{\sigma}}=\lambda(\mathbf{D}: \mathbf{E}) \mathbf{E}+2 G\left(\dot{\boldsymbol{\varepsilon}}-\dot{\boldsymbol{\varepsilon}}^{p}-\dot{\mathbf{p}}\right) \\
& \dot{\boldsymbol{\varepsilon}}^{p}=\Gamma_{1} \boldsymbol{\sigma}_{d}-\Gamma_{2} \frac{\partial F}{\partial \mathbf{p}} \\
& \dot{\mathbf{p}}=\Gamma_{2} \boldsymbol{\sigma}_{d}-\Gamma_{3} \frac{\partial F}{\partial \mathbf{p}} \\
& \dot{\delta}=-\Gamma_{4} \frac{\partial F}{\partial \delta} \\
& \frac{F}{F_{m}}=\frac{p^{2}}{2}-\frac{p^{2}}{2 \delta}+c_{1} p+c_{2} \ln \left(c_{3}+c_{4} p+p^{2}\right)-\frac{\boldsymbol{\sigma}_{d}: \mathbf{p}}{2 G} \\
& \boldsymbol{\sigma}_{d}=\sigma-\sigma_{s}, \sigma_{s}=\frac{1}{3}(\boldsymbol{\sigma}: \mathbf{E}) \mathbf{E}
\end{aligned}
$$


where: $\Gamma_{1}=\frac{l_{3}}{l_{1} l_{3}-l_{2}^{2}}, \Gamma_{2}=\frac{l_{2}}{l_{1} l_{3}-l_{2}^{2}}, \Gamma_{3}=\frac{l_{1}}{l_{1} l_{3}-l_{2}^{2}}, \Gamma_{4}=\frac{1}{l_{4}} ; \mathbf{E}$ - the unit tensor, $\sigma_{d}$ and $\sigma_{s}$ - the deviator and spherical parts of the stress tensor; $F_{m}, c_{1}-c_{4}-$ potential approximation constants, $G-$ shear modulus; $\lambda$ - the first parameter Lame; $p=\sqrt{\mathbf{p}: \mathbf{p}}$.

In general, kinetic coefficients depend on state parameters and are representable as follows:

$$
\Gamma_{i}=\frac{1}{G \tau_{i}}
$$

where $\tau_{i}$ - the characteristic relaxation times, which in general can be represented as dependencies:

$$
\tau_{i}=\tau_{i}^{0} \exp \left(-\frac{U_{i}\left(\boldsymbol{\sigma}, \boldsymbol{\varepsilon}, \dot{\varepsilon}, \boldsymbol{\varepsilon}^{p}, \dot{\boldsymbol{\varepsilon}}^{p}, \mathbf{p}, \dot{\mathbf{p}}, \delta, T\right)}{k T}\right),
$$

where: $k$ is the Boltzmann constant, $U_{i}(\cdot)$ is the characteristic activation energy. The hypothesis is accepted that for the studied processes the relaxation times have the following dependencies:

$$
\begin{aligned}
& \tau_{i}=\tau_{i}^{0} \exp \left(-\frac{U_{i} \dot{\varepsilon}_{0}^{n_{i}}-U_{0}}{k T}\right), i=1,2,3 \\
& \tau_{4}=\tau_{4}^{0} \exp \left(-\frac{U_{4} \dot{p}_{0}^{n_{\delta}}-U_{0}}{k T}\right)
\end{aligned}
$$

where: $\dot{\varepsilon}_{0}=\frac{\sqrt{\frac{2}{3} \dot{\varepsilon}: \dot{\varepsilon}}}{\dot{\varepsilon}_{c}}, \dot{\varepsilon}_{c}=1 \mathrm{~s}-1$ is the dimensionless factor; $U_{0}=k T, U_{i}, i=1 \ldots 3$ are the constants that have the meaning of the characteristic energy of overcoming barriers, after which new relaxation mechanisms are activated; $\dot{p}_{0}=\frac{\sqrt{\dot{\mathrm{p}}: \dot{\mathbf{p}}}}{\dot{\varepsilon}_{c}}$; $n_{1}, n_{2}, n_{3}, n_{\delta}$ - constants, characterizing the rate sensitivity of the material. The meaning of this hypothesis is that the physicomechanical characteristics of the material depend on the strain rate, and the evolution of damage under cyclic loading with small stress amplitudes depends only on the parameter, which in this case can be interpreted as the growth rate of a fatigue crack, related to its characteristic scale.

Assuming a weak strain rate sensitivity of the relaxation times, we can expand the expression (20) into a Taylor series with an accuracy of the second term:

$$
\exp \left(\frac{U_{i} \dot{\varepsilon}_{0}^{n_{i}}-U_{0}}{k T}\right) \approx 1+\frac{U_{i} \dot{\varepsilon}_{0}^{n_{i}}-U_{0}}{k T}=1+\frac{U_{i} \dot{\varepsilon}_{0}^{n_{i}}}{k T}-\frac{U_{0}}{k T}=\frac{U_{i} \dot{\varepsilon}_{0}^{n_{i}}}{k T}
$$

then we get:

$$
\tau_{i}=\tau_{i}^{0} \frac{1}{\frac{U_{i} \dot{\varepsilon}_{0}^{n_{i}}}{k T}}=\frac{\tau_{i}^{0} k T}{U_{i} \dot{\varepsilon}_{0}^{n_{i}}}=\frac{\tau_{i}^{U}}{\dot{\varepsilon}_{0}^{n_{i}}}
$$

Similarly, for the expression (21): 


$$
\tau_{4}=\tau_{4}^{0} \exp \left(-\frac{U_{4} \dot{p}_{0}^{n_{\delta}}-U_{0}}{k T}\right)=\tau_{4}^{0} \frac{1}{\exp \left(\frac{U_{4} \dot{p}_{0}^{n_{\delta}}-U_{0}}{k T}\right)} \approx \tau_{4}^{0} \frac{1}{1+\frac{U_{4} \dot{p}_{0}^{n_{\delta}}-U_{0}}{k T}}=\frac{\tau_{4}^{0} k T}{U_{4} \dot{p}_{0}^{n_{\delta}}}=\frac{\tau_{4}^{U}}{\dot{p}_{0}^{n_{\delta}}}
$$

Then the kinetic coefficients will have the following form:

$$
\Gamma_{i}=\frac{\dot{\varepsilon}_{0}^{n_{i}}}{G \tau_{i}^{U}}, i=1 \ldots 3, \Gamma_{4}=\frac{\dot{p}_{0}^{n_{\delta}}}{G \tau_{4}^{U}}
$$

We introduce the notation:

$$
\Gamma_{1}=\Gamma_{\sigma} \dot{\varepsilon}_{0}^{n_{1}}, \Gamma_{2}=\Gamma_{p \sigma} \dot{\varepsilon}_{0}^{n_{2}}, \Gamma_{3}=\Gamma_{p} \dot{\varepsilon}_{0}^{n_{3}}, \Gamma_{4}=\Gamma_{\delta} \dot{p}_{0}^{n_{\delta}}, n_{\varepsilon}=n_{1}+n_{2}, n_{p}=n_{2}+n_{3}
$$

The final determining equation is the fracture criterion. In the framework of the proposed model, we can enter a criterion based on the structural scaling parameter:

$$
\delta \rightarrow 0
$$

The meaning of this criterion is that the percentage ratio of the volume of material occupied by defects tends to $100 \%$, which can be achieved by tending to zero the distance between defects or tending to infinity of the size of defects. The second option is impossible, and the first has a clear physical meaning. In the calculation, the condition (22) is not realizable due to the instability, as it is, therefore, we can replace it with a softer one:

$$
\delta \leq \delta_{f}
$$

where $\delta_{f} \approx 0.4$ is the critical value [22], after which the avalanche-like growth of defects begins and the material is considered destroyed. This value is universal for all plastic materials for the proposed model. Critical value $\delta=\delta{ }_{f}$ corresponds to critical value $p_{c}$.

\section{Model Parameter Identification}

The identification of the parameters of the constructed model (15)-(19) was carried out in the uniaxial case:

$$
\begin{aligned}
& \dot{\sigma}=\lambda \dot{\varepsilon}+2 G\left(\dot{\varepsilon}-\dot{\varepsilon}^{p}-\dot{p}\right) \\
& \dot{\varepsilon}^{p}=\dot{\varepsilon}_{0}^{n_{\varepsilon}}\left(\Gamma_{\sigma} \sigma-\Gamma_{p \sigma} \frac{\partial F}{\partial p}\right) \\
& \dot{p}=\dot{\varepsilon}_{0}^{n_{p}}\left(\Gamma_{p \sigma} \sigma-\Gamma_{p} \frac{\partial F}{\partial p}\right) \\
& \dot{\delta}=-\dot{p}_{0}^{n_{\delta}} \Gamma_{\delta} \frac{\partial F}{\partial \delta} \\
& \frac{F}{F_{m}}=\frac{p^{2}}{2}-\frac{p^{2}}{2 \delta}+c_{1} p+c_{2} \ln \left(c_{3}+c_{4} p+p^{2}\right)-\frac{\sigma p}{2 G}
\end{aligned}
$$


where: $\dot{\varepsilon}, \sigma, \dot{\varepsilon}^{p l}, p$ are the corresponding components of the tensors $\dot{\varepsilon}, \sigma, \dot{\varepsilon}^{p}, \mathbf{p}$.

At the first stage of identification, the problem of minimizing the discrepancy between the experimental and calculated stress-strain diagrams is solved. This determines the parameters $\Gamma_{\sigma}, \Gamma_{p \sigma}, \Gamma_{p}$; dimensionless strain rate $\dot{\varepsilon}_{0}=1$ (static loading), $\delta=$ const $=1.15$.

At the second stage, using the known values $\Gamma_{\sigma}, \Gamma_{p \sigma}, \Gamma_{p}$ the problem of minimizing the discrepancy between the experimental and calculated stress-strain diagrams at different strain rates is solved. This determines the parameters $n_{\varepsilon}$ and $n_{p}, \delta=$ const $=1.15$. An illustration of optimization problem solving is presented in Tab. 3 .

\begin{tabular}{ccc}
\hline $\begin{array}{c}\text { Strain rate, } \\
\mathrm{s}^{-1}\end{array}$ & $\begin{array}{c}\text { Yield strength, } \mathrm{MPa} \\
\text { (the experiment, }[25,26])\end{array}$ & $\begin{array}{c}\text { Yield strength, } \\
\mathrm{MPa} \text { (the calculation) }\end{array}$ \\
0.0001 & 165 & 166 \\
520 & 175 & 176 \\
1210 & 210 & 213 \\
\hline
\end{tabular}

Table 3: The yield strength in the experiment $[25,26]$ and the calculation at different strain rates.

The final step in identifying the parameters of the model is to determine the values of $\Gamma_{\delta}$ and $n_{\delta}$ that are found using experimental fatigue loading data. Two characteristic points on the Wöhler curve were selected: (300,000 cycles, $190 \mathrm{MPa})$ and $(300,000,000$ cycles, $156 \mathrm{MPa})$. Parameters $\Gamma_{\delta}$ and $n_{\delta}$ were chosen in such a way that at the loading amplitude of 190 $\mathrm{MPa}$ the material was destroyed after 300,000 cycles, and at $156 \mathrm{MPa}$ - after 300,000,000 cycles, respectively. The frequency of loading in this case was equal to $20000 \mathrm{~Hz}$, as in the experiment.

Thus, the complete set of constants for the AlMg6 alloy is as follows:

Constants known from literature [24]: $\varrho=2670 \mathrm{~kg} / \mathrm{m} 3, \lambda=41 \mathrm{GPa}, G=27 \mathrm{GPa}$.

Constants that were defined: $\Gamma_{\sigma}=529(\mathrm{~Pa} \cdot \mathrm{s})^{-1}, \Gamma_{p \sigma}=38.5(\mathrm{~Pa} \cdot \mathrm{s})^{-1}, \Gamma_{p}=2.9(\mathrm{~Pa} \cdot \mathrm{s})^{-1}, \Gamma_{\delta}=3.46(\mathrm{~Pa} \cdot \mathrm{s})^{-1}, n_{\varepsilon}=n_{p}=0.967$, $n_{\delta}=1.985$.

\section{Numerical simulation results}

A numerical study of the behavior of a real structure and even a laboratory specimen in the very high cycle fatigue mode is not possible due to the requirement of huge computational and time resources. Therefore, instead of solving the boundary value problem, the problem of loading a representative material volume (24)-(28) with loading conditions in the form of applied cyclic stresses (29) with amplitude $\sigma_{A}$ and frequency $v=20000 \mathrm{~Hz}$, as well as initial conditions (21) is considered.

$$
\begin{aligned}
& \sigma(t)=\sigma_{A} \sin (2 \pi v t) \\
& \left.p\right|_{t=0}=0,\left.\sigma\right|_{t=0}=0,\left.\varepsilon^{p}\right|_{t=0}=0,\left.\delta\right|_{t=0}=\delta_{0}=1.15
\end{aligned}
$$

The loading condition (19) can be written in terms of strain:

$$
\varepsilon(t)=\varepsilon\left(\sigma_{A}\right) \sin (2 \pi v t)
$$

where $\varepsilon(\sigma)$ is the function that converts stresses into strains for AlMg6 alloy. Differentiating (31) by time, we get:

$$
\dot{\varepsilon}(t)=\varepsilon\left(\sigma_{A}\right) 2 \pi v \cos (2 \pi v t)
$$

The numerically constructed Wöhler curve and its comparison with experimental data of authors and experiment [27] with similar experiment conditions (AlMg- $6, \mathrm{R}=-1$, frequency $10 \mathrm{kHz}$ ) are presented in Fig. 6. 


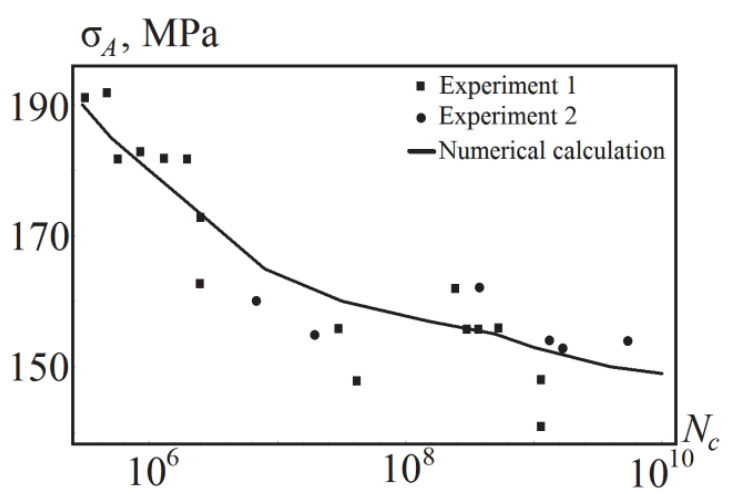

Figure 6: Wöhler curve for AlMg6 alloy. Solid line - numerical calculation, points - experimental data of the authors, squares experiment [27] with conditions: frequency $10 \mathrm{kHz}, \mathrm{R}=-1$.

Due to the amplitudes of the applied stresses below the yield strength, the material is macroscopically in the elastic region, and the applied deformations can be considered small. Then expression (24) can be rewritten in the form:

$$
\sigma=\lambda \varepsilon+2 G\left(\varepsilon-\varepsilon^{p}-p\right)
$$

Since macroplastic strain is not observed, then in the very high cycle fatigue mode, the parameter $\mathrm{p}$ is responsible for the accumulation of damage and destruction. The growth of defects leads to a gradual change in the amplitude of the applied voltages, and consequently to a change in the amplitude of the applied displacements, since for some displacement amplitude $U_{A}$ in the loading machine is always assigned $\sigma_{A}$ :

$$
\sigma_{A}=\lambda \varepsilon\left(\sigma_{A}\right)+2 G\left(\varepsilon\left(\sigma_{A}\right)-p\right)
$$

After reaching the parameter $\mathrm{p}$ of some critical value $\mathrm{pc}$ (which corresponds to $\delta_{f} \approx 0,4$ ), an avalanche-like growth of defects occurs, leading to the destruction of the material, which in the experiment corresponds to an avalanche-like change in the amplitude of the second harmonic of displacements (Fig. 7.).

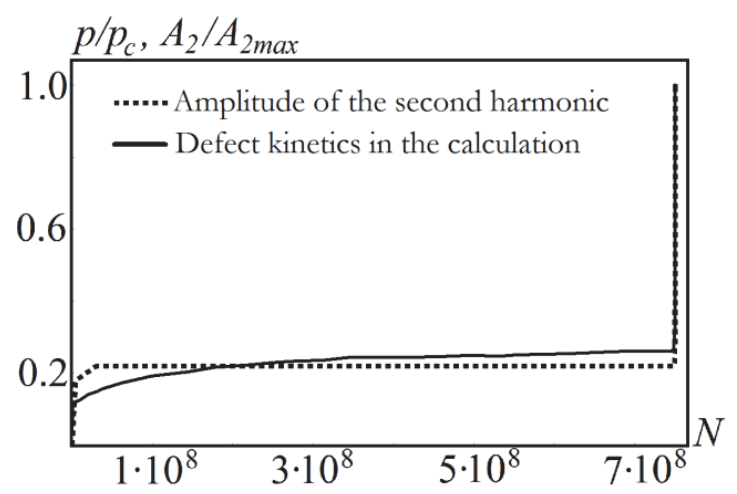

Figure 7: Defect kinetics (dashed line) in the calculation and change in the amplitude of the second harmonic of displacements in the experiment (solid line)

In fig. 7 shows the normalized curves of variation of the parameter p, referred to pc and the amplitude of the second harmonic of displacements, referred to its maximum value. Figure 7 shows the experimental data corresponding to the preloaded specimen. According to the proposed model, the change in structure due to preloading is described in terms of the parameter $\delta$. Namely, a smaller value of the initial condition $\delta_{0}$ corresponds to the preloaded specimen. To obtain the dotted curve (Fig. 7), the Eqns. (24-29, 30, 32) was solved with the initial condition $\delta_{0}=1.02$.

Thus, the proposed model is able to adequately describe the behavior of the material in the modes of high- and very high cycle fatigue. Based on the results of numerical simulation, we can conclude that the change in the amplitude of the 
second harmonic of displacements in the experiment gives adequate information about the accumulation of damage in the material.

\section{CONCLUSION}

A $\mathrm{n}$ analysis of the free end oscillations during gigacyclic tests showed a significant increase in the second harmonic amplitude and nonlinearity coefficient during the formation of fatigue cracks. In conjunction with the simulation results, we can conclude that the deviation from the linear-elastic law ("anomaly of elastic compliance") is due to the accumulation of defects in the material, and the avalanche-like growth of the second harmonic displacement corresponds to the avalanche-like growth of defects and the formation of a macroscopic crack. Thus, it is possible to judge the accumulation of damage, including - in the amount of material based on the acoustic signal. Using the proposed equations, it can to predict the fatigue life of the material and identify internal defects and cracks before macroscopic destruction occurs.

\section{ACKNOWLEDGMENTS}

A uthors thank Prof. Valery Matveyenko for the proposal to present the paper in the issue. This work was supported by the Russian Science Foundation, grant number 18-72-00142.

\section{REFERENCES}

[1] Botvina, L.R. (2004) Gigacycle fatigue is a new problem in physics and mechanics of destruction // Factory Laboratory. Diagnostics of materials 70(4), pp. 41. (In Russian).

[2] Bathias, C., Paris, P.C. (2005), Gigacycle Fatigue in Mechanical Practice, Marcel Dekker Publisher Co., pp. 328.

[3] Oborin, V., Bannikov, M., Naimark, O., Palin-Luc, T. (2010). Scaling invariance of fatigue crack growth in gigacycle loading regime, Technical Physics Letters, 36 (11), pp. 1061-1063. DOI: 10.1134/S106378501011026X.

[4] Cowles, B.A. (1996). High cycle fatigue in aircraft gas turbines - an industry perspective, International Journal of Fracture 80, pp.147-163. DOI: 10.1007/BF00012667.

[5] Shanyavsky, A.A. (2007) Simulation of fatigue damage of metals. Synergetic in aviation, Ufa: Monograph LLC. (In Russian).

[6] Nicholas, T. (2006). High Cycle Fatigue. A Mechanics of Material Perspective, Elsevier., pp. 641.

[7] Peters, J.O., Ritchie, R.O. (2000). Influence of foreign object damage on crack initiation and early crack growth during high-cycle fatigue of Ti-6Al-4V, Eng. Fract. Mech. 67, pp. 193-207. DOI: 10.1016/S0013-7944(00)00045-X.

[8] Spanrad, S., Tong, J. (2011). Characterisation of foreign object damage (FOD) and early fatigue crack growth in laser shock peened Ti-6Al-4V aerofoil specimens, Materials Science and Engineering A, 528, pp. 2128-2136.

DOI: 10.1016/j.msea.2010.11.045.

[9] Oakley, S.Y., Nowell, D. (2007) Prediction of the combined high- and low-cycle fatigue performance of gas turbine blades after foreign object damage, International Journal of Fatigue, 29, pp. 69-80. DOI: 10.1016/j.ijfatigue.2006.02.042.

[10] Chen, Xi (2005). Foreign object damage on the leading edge of a thin blade, Mechanics of Materials, 37, pp. 447-457. DOI: https://doi.org/10.1016/j.mechmat.2004.03.005.

[11] Nowell, D., Duó, P., Stewart, I.F. (2003). Prediction of fatigue performance in gas turbine blades after foreign object damage, International Journal of Fatigue, 25, pp. 963-969. DOI: 10.1016/S0142-1123(03)00160-9.

[12] Mughrabi, H. (2015). Microstructural mechanisms of cyclic deformation, fatigue crack initiation and early crack growth, Philosophical Transactions of the Royal Society A: Mathematical, Physical and Engineering Sciences, 373(2038), 20140132. DOI: 10.1098/rsta.2014.0132.

[13] Zhang, Li-Li, et al. (2016). On the formation mechanisms of fine granular area (FGA) on the fracture surface for high strength steels in the VHCF regime, International Journal of Fatigue, 82, pp. 402-410.

DOI: 10.1016/j.ijfatigue.2015.08.021. 
[14] Oborin, V., Sokovikov, M., Bilalov, D., \& Naimark, O. (2016). Multiscale study of morphology of the fracture surface aluminum-magnesium alloy with consecutive dynamic and gigacycle loading, Procedia Structural Integrity, 2, pp. 10631070. DOI: $10.1016 /$ j.prostr.2016.06.136.

[15] Froustey, C., Naimark, O., Bannikov, M., Oborin, V. (2010). Microstructure scaling properties and fatigue resistance of pre-strained aluminium alloys (part 1: AlCu alloy), European Journal of Mechanics A/Solids, 29, pp. 1008-1014. DOI: 10.1016/j.euromechsol.2010.07.005.

[16] Oborin, V.A., Bayandin, Yu. V., Bilalov, D. A., Sokovikov, M. A., Chudinov, V. V, Naimark, O. B. (2018). Self-similar laws of damage development and evaluation of the reliability of alloys D16T and AMg6 under combined dynamic and gigacycle loading, Phys. Mezomekh, 21(6), pp. 135-145 DOI: 10.1134/S1029959919020048.

[17] John H. Cantrell, William T. Yost (2001) Nonlinear ultrasonic characterization of fatigue microstructures, Int. J. of Fatigue, 23, pp.487-490. DOI: 10.1016/S0142-1123(01)00162-1.

[18] Kumar, A., Torbet, J.C. Pollock, M.T., Jones, W.J. (2010). In situ characterization of fatigue damage evolution in a cast $\mathrm{Al}$ alloy via nonlinear ultrasonic measurements, ActaMaterialia, 58(6), pp. 2143-2154.

DOI: $10.1016 /$ j.actamat.2009.11.055

[19] Kumar A. et al. (2011) In situ damage assessment in a cast magnesium alloy during very high cycle fatigue. ScriptaMaterialia, 64(1), pp.65-68. DOI: 10.1016/j.scriptamat.2010.09.008

[20] Nazarov A.A., (2018). Nonequilibrium grain boundaries in bulk nanostructured metals and their recovery under the influences of heating and cyclic deformation. Review, Letters on Materials 8 (3), 2018 pp. $372-381$ (in Russian). DOI: $10.22226 / 2410-3535-2018-3-372-381$

[21] W. Li, H. Cui, W. Wen, X. Su, C. C. Engler-Pinto Jr.: (2015). In situ Nonlinear Ultrasonic for Very High Cycle Fatigue Damage Characterization of a Cast Aluminum Alloy. Materials Science and Engineering A, 645, pp. $248-254$. DOI: $10.1016 /$ j.msea.2015.08.029.

[22] Bilalov, D.A., Bayandin, Yu.V., Naimark, O.B. (2018). Mathematical modeling of failure process of AlMg2.5 alloy during high- and very high cycle fatigue, Computational continuum mechanics. 11(3), pp. 323-334. (In Russian). DOI: 10.7242/1999-6691/2018.11.3.24.

[23] Naimark, O.B. (2003) Collective properties of defects ensembles and some nonlinear problems of plasticity and fracture, Physical mesomechanics, 6(4), pp. 39-63.

[24] Froustey C., Naimark O.B., Panteleev I.A., Bilalov D.A., Petrova A.N., Lyapunova E.A. (2017) Multiscale structural relaxation and adiabatic shear failure mechanisms, Physical Mesomechanics, 20(1), pp. 31-42. DOI: $10.1134 /$ S1029959917010039.

[25] Glushak, B.L., Ignatova, O.N., Pushkov, V.A., Novikov, S.A., Girin, A.S., Sinitsyn, V.A. (2000). Dynamic Deformation of Aluminum Alloy AMg-6 at Normal and Higher Temperatures, Journal of Applied Mechanics and Technical Physics., 41(6), pp. 1083-1086. DOI: 10.1023/A:1026662824249.

[26] Frolov, K.V. (2001). Mechanical Engineering. Encyclopedia. Volume II-3: Non-ferrous metals and alloys. Composite metallic materials. Moscow, MechanicalEngineering, 880 p. (In Russian).

[27] Yakovleva, T. Yu., Matokhnyuk, L.E. (2004). Prediction of fatigue metal resistance characteristics at different loading frequencies, Strength problems, 4, pp. 145-155. (In Russian). 